\title{
Overlap Syndrome of Functional Dyspepsia and Irritable Bowel Syndrome - Are Both Diseases Mutually Exclusive?
}

\author{
Hidekazu Suzuki* and Toshifumi Hibi \\ Division of Gastroenterology and Hepatology, Department of Internal Medicine, Keio University School of Medicine, Tokyo, Japan
}

Among functional gastrointestinal (GI) disorders, functional dyspepsia (FD) and irritable bowel syndrome (IBS) are important to public health around the world and are frequently encountered in general practice. Upper Gl symptoms such as heartburn, postprandial fullness, early satiety, epigastric pain or burning and lower Gl symptoms such as constipation and diarrhea often coexist. Although the prevalence of FD-IBS overlap would be influenced by the selection of the study population, the overlap rate of FD-IBS could be in the range of $11 \%-27 \%$. Specifically, FD-IBS overlap is associated with more severe symptoms than FD alone or IBS alone. Since clinical overlap, especially FD-IBS overlap, is very common, the 2 syndromes should not be treated in a mutually exclusive fashion.

(J Neurogastroenterol Motil 2011;17:360-365)

Key Words

Dyspepsia; Epigastric pain syndrome; Health-related quality of life; Irritable bowel syndrome; Postprandial distress syndrome

\section{Introduction}

Epidemiological studies suggest a considerably high rate of overlap between functional dyspepsia (FD) and irritable bowel syndrome (IBS). According to the recent systematic review and metanalysis, ${ }^{1}$ the prevalence of IBS in subjects with dyspepsia is $37 \%(95 \% \mathrm{CI}, 30 \%-45 \%)$ as compared to $7 \%$ (95\% CI, $5 \%-$ $10 \%)$ in those without. The pooled odds ratio for IBS in subjects with dyspepsia was 8 (95\% CI, 5.74-11.16) as compared to that in those without dyspepsia. However, the original studies used an older classification such as Manning's, Rome I or II, than Rome III and also did not exclude organic diseases, possibly resulting in the contamination with peptic ulcer diseases or reflux esophagitis.

\section{Prevalence of Overlap Between \\ Functional Dyspepsia and Irritable Bowel Syndrome}

There are a few evaluations of overlap between FD and IBS

Received: June 29, 2011 Revised: July 9, 2011 Accepted: July 14, 2011

(c) This is an Open Access article distributed under the terms of the Creative Commons Attribution Non-Commercial License (http://creativecommons. org/licenses/by-nc/3.0) which permits unrestricted non-commercial use, distribution, and reproduction in any medium, provided the original work is properly cited.

${ }^{*}$ Correspondence: Hidekazu Suzuki, MD, PhD

Associate Professor, Division of Gastroenterology and Hepatology, Department of Internal Medicine, Keio University School of Medicine, 35 Shinanomachi, Shinjuku-ku, Tokyo 160-8582, Japan

Tel: +81-3-5363-3914, Fax: +81-3-5363-3967, E-mail: hsuzuki@a6.keio.jp

Financial support: This work was supported by a Health and Labour Sciences Research Grant (Clinical Research Promotion No. 47) for Research on Health Technology Assessment (to H.S.), a grant (11035231-000061) from the JSPS Bilateral Joint Projects with Belgium, a grant from the Smoking Research Foundation (to H.S.), the Keio Gijuku Academic Development Fund (to H.S.) and a Nateglinide Memorial Toyoshima Research and Education Fund (to H.S.).

Conflicts of interest: The contents of this article had been lectured as a special lecture in the 12th Recent Update of Neurogastroenterology and Motility Symposium, held on June 11, 2011 at Novotel Ambassador Busan Hotel. 
based on the Rome III classification. Kaji et $\mathrm{al}^{2}$ conducted a cross-sectional study to examine the prevalences of gastroesophageal reflux, FD and IBS, their overlap rates, and the health-related quality of life (HRQOL) for each disease and each overlap syndrome, as compared with the corresponding values in healthy controls in a Japanese health check-up population. Of the 2,680 eligible subjects, 269 (10.0\%) were diagnosed as having FD, and 381 (14.2\%) as having IBS. Overlaps between FD and IBS were found in 92 patients (3.4\%). Overlap significantly worsened the HRQOL in most domains, except in the "role emotional" domain. The HRQOL was particularly poor in the mental component summary for overlapping IBS. ${ }^{2}$ On the other hand, Nakajima et $\mathrm{al}^{3}$ conducted a survey in a general gastroenterology outpatient clinic of 1,378 consecutive patients. Among them, 29 (2.1\%) were diagnosed as having FD, and $33(2.4 \%)$ as IBS. Overlaps between FD and IBS were found in $12(0.9 \%)$.

Wang et $\mathrm{al}^{4}$ investigated 3,014 patients who responded to their questionnaires (male:female $=47.2: 52.8$, response rate $89 \%$ ) at a general gastroenterology outpatient clinic. FD-IBS overlap was observed in 151 (5.0\%) patients, while 457 (15.2\%) and $329(10.9 \%)$ were classified as having FD alone and IBS alone, respectively. ${ }^{4}$ As compared with that in the non-IBS cohorts, the OR of having FD among IBS was 2.09 (95\% CI, 1.68-2.59). Patients with FD-IBS overlap had higher severity scores for the symptom of postprandial fullness $(2.4 \pm 1.5$ vs 1.7 $\pm 1.6, P<0.001)$ and overall FD symptoms $(6.7 \pm 2.9$ vs $5.8 \pm$ 2.8, $P<0.05)$ than those with FD alone. The only independent risk factor for FD-IBS overlap versus $\mathrm{FD}$ alone was the presence of postprandial fullness (OR, 2.67; 95\% CI, 1.34-5.31). ${ }^{4}$

Lee et $\mathrm{al}^{5}$ investigated the differences in depressive mood and quality of life (QOL) among Korean patients with FD, IBS and FD-IBS overlap diagnosed by the Rome III definition. According to their report, ${ }^{5}$ out of 279 subjects, 70 (25.1\%) and 124 (44.4\%) were diagnosed as having FD and IBS, respectively. Patients with FD-IBS overlap and those with FD alone showed higher Beck Depressive Inventory scores than normal subjects $(P$ $<0.001$ and $P=0.02$, respectively), whereas those with IBS alone showed no differences in scores from normal subjects $(P=$ 0.17). All of the SF-36 (the 36-item Short Form general health survey) subscores of the FD-IBS overlap cohorts were significantly lower than those in normal subjects $(P<0.05)$. Depressive mood was significantly related to FD and FD-IBS overlap, but not to IBS alone. Patients with FD-IBS overlap had a worse QOL than patients with FD alone or IBS alone. ${ }^{4}$ Furthermore, according to the recent report by Park, ${ }^{6}$ the sensi- tivity and specificity of the Rome III classification in discriminating functional gastrointestinal disorders (FGIDs) from organic diseases of the upper gastrointestinal (GI) tract were $60 \%$ and $53 \%$, respectively, while the values of the lower GI tract were $80 \%$ and $50 \%$, respectively, partially supporting the use of the Rome III criteria in Korea. ${ }^{5}$

Data on the impact of FD on the HRQOL in the general population are scarce. Aro et $\mathrm{al}^{7}$ explored the impact of $\mathrm{FD}$ based on the Rome III classification on the HRQOL in the general population. Among 1,001 cohorts, 202 (20\%) individuals reported uninvestigated dyspepsia (UID), and 157 (16\%) reported FD. FD-IBS overlap had a significant impact on bodily pain $(P$ $<0.01)$ and general health $(P<0.05)^{7}$

Although Hori et $\mathrm{al}^{8}$ used the Rome II criteria, they examined concurrent GI symptoms in FD and IBS in a total of 186 college students who filled out a questionnaire administered to determine whether they had UID or IBS. The diagnosis of UID, IBS and UID + IBS overlap was made in 12 (6.7\%), 40 $(22.1 \%)$ and $8(4.4 \%)$ patients, respectively and a significant prevalence of UID + IBS overlap was observed (66.7\% IBS in UID; $20.0 \%$ UID in IBS). ${ }^{8}$ Although Corsetti et $\mathrm{al}^{9}$ also used Rome II criteria for the diagnosis of FD in their questionnaire survey of 309 consecutive FD patients to assess the dyspepsia and IBS symptom patterns, $54 \%$ of the patients had FD alone, whereas $46 \%$ had FD + IBS. FD-IBS overlap patients were more likely to be female $(75 \%$ vs $60 \%, P<0.01)$ and to have greater weight loss $(5.4 \pm 0.6$ vs $3.5 \pm 0.4 \mathrm{~kg}, P<0.05)$. Coexisting IBS did not increase the risk of dyspepsia, however, the overall symptom severity was significantly higher in the patients with FD-IBS overlap $(12.4 \pm 0.4$ vs $9.8 \pm 0.3, P<0.01)$. FD-IBS overlap patients had a lower threshold for first perception $(2.9 \pm$ 0.3 vs $3.8 \pm 0.3 \mathrm{mmHg}, P<0.05)$ and for discomfort $(7.9 \pm$ 0.4 vs $9.5 \pm 0.5 \mathrm{mmHg}, P<0.05)$ and a greater prevalence of hypersensitivity to gastric distention ( $44 \%$ vs $28 \%, P<0.05)$. ${ }^{9}$

Recently, we performed a web-based survey comprised of Rome III criteria for FD, the Gastrointestinal Symptom Rating Scale, and questions to determine demographic information among subjects registered for Japanese clinical trial programs. ${ }^{10}$ Cluster analysis revealed 3 distinct clusters: cluster associated with diarrhea, cluster associated with constipation and cluster associated with neither diarrhea nor constipation. Cluster associated with constipation and cluster associated with diarrhea were significantly linked to the presence of FD, suggesting that FD was more prevalent among participants with bowel symptoms than in those without. Furthermore, FD patients with bowel 
symptoms had more severe dyspepsia symptoms than those without.

Although GI symptoms are quite common in the general population, different methods for the survey show different epidemiologies, and the effects of psychosocial and behavioral factors on the symptoms have been studied, mainly by subgroup analysis. According to the Japanese questionnaire survey focusing on GI symptoms and the psycho-behavioral background in members of a registered panel via e-mail and postal mail, despite the difference in the prevalence of GI symptoms, that is, $47 \%$ in the electronic survey and $25 \%$ in the postal one, similar proportions of symptom subtypes and patterns of overlaps were obtained with such 2 methods. ${ }^{11}$ While $56 \%$ were diagnosed as having $\mathrm{FD}$, and $58 \%$ as having IBS in the electronic survey, $57 \%$ were diagnosed as having FD, and $55 \%$ as having IBS in the postal one. FD-IBS overlap was found in $24 \%$ in the electronic survey and in $23 \%$ in the postal one. Subjects who have higher scores for psycho-behavioral problems had a higher prevalence of FD and IBS symptoms. The data suggest that psycho-behavioral conditions may affect the development of functional GI symptoms, regardless of the subtype of GI disorders, and can explain the high proportion of overlap among the subtypes. ${ }^{11}$

Taken together, the above-mentioned prevalence of FD-IBS overlap would be altered depending on the selected study population. The rate of FD-IBS overlap could be in the same range, such as $11.4 \%,{ }^{6} 16.5 \%,{ }^{2} 23.8 \%,{ }^{4} 24.0 \%^{3}$ and $27.6 \%{ }^{5}$ (Table).

\section{Influence of Each Subtype of Functional Dyspepsia or Irritable Bowel Syndrome on the Presence of Functional Dyspepsia- Irritable Bowel Syndrome Overlap}

A general definition of $\mathrm{FD}$, to be used mainly for clinical

Table. Prevalence of Overlap Between Functional Dyspepsia and Irritable Bowel Syndrome

\begin{tabular}{lrrr}
\hline & $\begin{array}{c}\text { FD alone } \\
(\mathrm{n}[\%])\end{array}$ & $\begin{array}{c}\text { IBS alone } \\
(\mathrm{n}[\%])\end{array}$ & $\begin{array}{c}\text { FD-IBS overlap } \\
(\mathrm{n}[\%])\end{array}$ \\
\hline Wang et al $^{4}$ & $306(48.2)$ & $178(28.0)$ & $151(23.8)$ \\
Nakajima et al $^{3}$ & $17(34.0)$ & $21(42.0)$ & $12(24.0)$ \\
Kaji et al $^{2}$ & $177(31.7)$ & $289(51.8)$ & $92(16.5)$ \\
Lee et al $^{5}$ & $28(18.4)$ & $82(53.9)$ & $42(27.6)$ \\
Park $^{6}$ & $72(43.1)$ & $76(45.5)$ & $19(11.4)$ \\
\hline
\end{tabular}

FD, functional dyspepsia; IBS, irritable bowel syndrome. purposes, and although further research on more specific definitions is ongoing, is provided under category $\mathrm{B} 1$ : functional dyspepsia (FD) in Rome III classification. However, particularly for pathophysiological and therapeutic approach, it is recommended in Rome III that new entities of meal-induced dyspeptic symptoms as postprandial distress syndrome (PDS): Bla, and epigastric pain and burning as epigastric pain syndrome (EPS): B1b, should be used. ${ }^{12}$ These 2 subcategories such as PDS and EPS seem very useful in clinical practice as well as in the investigative settings. According to the report by Wang et al, ${ }^{4}$ more patients with PDS alone had IBS than those with EPS alone. Patients with FD-IBS overlap were more likely to be classified as the PDS + EPS subtype, had more frequent presence of the postprandial fullness symptom, one of major symptoms of PDS $(P<0.001)$, and overall FD symptom $(P<0.01)$ than those with FD alone. Furthermore, patients with FD-IBS overlap were less likely to be classified as the EPS alone $(P<0.01)$ compared to those classified as FD alone. By the multivariate logistic regression analysis, only the presence of postprandial fullness (OR, 2.67; 95\% CI, 1.34-5.31; $P<0.01$ ) had a statistically significant and independent effect on the probability of FD-IBS overlap. On the other hand, subtypes of IBS did not differ between patients with constipation type-IBS (IBS-C) and diarrhea-type IBS (IBS-D). In addition, neither IBS-D (OR, 1.44; 95\%CI, $0.93-2.22 ; P=0.10)$ nor mixed-type IBS (IBS-M) (OR, 1.62; 95\% CI, 0.77-3.40; $P=0.21$ ) was identified as risk factor for FD-IBS overlap versus IBS alone in the multivariable analysis. Aro et $\mathrm{al}^{7}$ reported the data on the impact of PDS and EPS on QOL. The impact of PDS is statistically significant compared with controls except for Role Emotional and the results are consistently the same when analyzing for the possible confounders such as anxiety, depression and use of proton pump inhibitors. Patients with EPS had a statistically relevant impairment of HRQOL only in 2 domains such as Bodily Pain and Vitality compared with nondyspeptic cohorts. Namely, PDS seems to damage HRQOL in all SF-36 domains more than EPS.

In addition, non-erosive relux disease is more frequently overlapped with FD, especially with EPS and presents with significantly increased frequency of IBS. ${ }^{13}$

\section{Pathophysiology Leading to Functional Dyspepsia-Irritable Bowel Syndrome Overlap}

FD and IBS are common functional disorders without de- 
fined pathophysiology and are known as multifactorial syndromes. The pathophysiological factors for the generation of FD or IBS would be listed as visceral hypersensitivity, central abnormal deregulation for sensory perception, GI dysmotility, and abnormal alteration of the intestinal flora, GI inflammation, and psychosocial factors. Although such a mechanistic investigation is further preceded in the research field in IBS, the detail of pathophysiology in FD-IBS overlap has not been fully explored. However, as these 2 syndromes (FD and IBS) would have common causative factors, the high prevalence of FD-IBS overlap is considered to be easily acceptable.

Abnormalities of psychosocial or central nervous factors, which would affect the whole GI tract, could be a possible causative factor in the pathogenesis of FD-IBS overlap. Savas et $\mathrm{al}^{14}$ examined the 1-year prevalence of IBS and dyspepsia symptoms and their associations with depression, anxiety and post-traumatic stress disorder (PTSD) among women veterans receiving primary care at a Women's Clinic, Veteran Affairs (VA) Medical Center. They reported that women with IBS-dyspepsia overlap showed higher scores of anxiety (IBS: 24 vs $12, P<0.001$ and dyspepsia: 26 vs $12, P<0.001$ ), depression (IBS: 22 vs $11, P$ $<0.001$ and dyspepsia: 23 vs $11, P<0.001$ ) and PTSD (IBS: 87 vs $69, P<0.001$ and dyspepsia: 86 vs $69, P<0.0005)$, and age- and ethnicity-adjusted logistic regression analyses revealed a 3- to 46-fold increase in the OR of IBS and dyspepsia among women with depression, anxiety or PTSD. ${ }^{14}$ Although gastric sensorimotor dysfunction, psychosocial factors and somatization are all implicated in the development of FD symptoms according to the report by Van Oudenhove et al, ${ }^{15}$ symptom severity and weight loss in FD are determined by psychosocial factors (depression or history of abuse) and somatization, and to a lesser extent by gastric sensorimotor function, but not by FD-IBS overlap. On the other hand, Kindt et $\mathrm{al}^{16}$ in Belgium investigated the 5 -year evolution of symptoms in a clinical FD population to identify factors associated with the outcome and indicated that the dyspepsia symptom score (DSS) at the initial visit and trait anxiety were longitudinally associated with the DSS at follow-up, with a trend found for weight loss; depression, chronic fatigue and IBS at follow-up were cross-sectionally associated with DSS.

On the other hand, GI inflammation and altered immune responses could be another candidate as the cause of FD-IBS overlap. Patients with IBS show a greater degree of inflammatory cell infiltration than healthy controls. Mast cells and eosinophils interact with $\mathrm{T}$ lymphocytes and may alter the enteric nerve and smooth muscle functions. Examination of 48 IBS patients with either diarrhea or constipation, ${ }^{17} 12$ patients with microscopic colitis, 20 patients with ulcerative colitis, and 24 healthy controls, indicated that as compared to male IBS patients, female IBS had greater numbers of mast cells $(P=0.066)$, but lesser numbers of $\mathrm{CD} 3+$ and $\mathrm{CD} 8+\mathrm{T}$ cells $(P<0.01$ and $P<0.001$, respectively). Such mucosal mast cell infiltration in IBS patients was significantly associated with the frequency of abdominal bloating $(P<0.05)$ and with dysmotility-like dyspepsia $(P=$ $0.001)$, but not with ulcer-like dyspepsia. ${ }^{17}$ According to the report by Walker et al, ${ }^{18}$ intraepithelial lymphocytes in IBS-C were significantly increased $(P=0.005)$ and mast cells were significantly increased in the second part of the duodenum in IBS $(P$ $<0.001$ ), while eosinophils were significantly increased in the duodenal bulb and second part of the duodenum in FD $(P<$ $0.001)$, suggesting that duodenal mast cell hyperplasia is linked to IBS, and eosinophilia to FD.

Recently, it has been revealed that previous infectious gastroenteritis is often followed by postinfectious FD (PI-FD) ${ }^{19}$ or postinfectious IBS (PI-IBS). ${ }^{20}$ Porter et $\mathrm{al}^{21}$ recently conducted a matched, case-control study describing the epidemiology and risk determinants of IBS, functional constipation, functional diarrhea and dyspepsia using electronic medical encounter data in active-duty US military personnel, and demonstrated a significant association between infectious gastroenteritis and all FGIDs (OR, 2.64; $P<0.001$ ), with the highest frequency of functional diarrhea (OR, 6.28; $P<0.001)$ and IBS (OR, 3.72; $P<$ $0.001)$, and a moderate frequencies of functional constipation (OR, 2.15; $P<0.001)$ and FD (OR, 2.39; $P<0.001)$. According to Kindt et $\mathrm{al}^{22}$ and Suzuki ${ }^{23}, \mathrm{PI}-\mathrm{FD}$ is associated with persistent focal $\mathrm{T}$ cell aggregates, decreased CD4 + cells and increased macrophage counts surrounding the crypts, indicating an impaired ability of the immune system to terminate the inflammatory response after an acute insult.

Since the etiology of FD is still uncertain, it is not surprising that Helicobacter pylori, a major pathogen in the stomach, ${ }^{24-26}$ has been implicated in the pathophysiology of so called dyspepsia. Many trials reporting the efficacy of $H$. pylori eradication therapy for FD including non-ulcer dyspepsia have given conflicting results but there is a clear indication that $H$. pylori eradication treatment is effective in at least a subset of patients with FD or non-ulcer dyspepsia. ${ }^{27,28}$ The recent meta-analysis also suggests that $H$. pylori eradication at 12 months has a small but statistically significant benefit in the treatment of FD (relative risk [RR] of remaining dyspepsia with $H$. pylori eradication therapy $=0.91$; 95\% CI, 0.87-0.99). While statistically significant, the clinical 
significance of this finding is less clear because the effect is small, that is, $15 \mathrm{H}$. pylori-positive dyspeptic patients will need to be treated to achieve just one cure. ${ }^{29}$ On the other hand in Asia, Gwee et $\mathrm{al}^{30}$ conducted a double blind, randomized, placebo-controlled trial of $H$. pylori eradication for FD was conducted in Singapore population. Forty-one patients received active treatment consisting of a 1-week course of omeprazole $20 \mathrm{mg}$ once daily, clarithromycin $250 \mathrm{mg}$ twice daily and tinidazole $500 \mathrm{mg}$ twice daily whereas another 41 patients received matching placebo tablets. They reported on ITT analyses, that while symptom resolution was observed in $24 \%$ of patients on active treatment, only $7 \%$ was found on the placebo $(P=0.02 ; 95 \% \mathrm{CI}, 1.1-17.7)$. In addition, their report shows that among patients with $H$. pylori eradicated on active treatment the symptom resolution rate was $39 \%$, whereas it was $3 \%$ among patients in the placebo who had persistent $H$. pylori infection, ${ }^{30}$ suggesting that patients with FD in Asian population might benefit more from treatment for $H$. $p y$ lori infection.

On the aspects of pathophysiology of dyspepsia in $H$. pylori infection, we recently reported in mice that after long-term $H$. $p y$ lori infection, gastric emptying was significantly accelerated with a significant thickening of the muscular layers of the stomachs that was manifest in the hyperplasia of myocytes. ${ }^{31} \mathrm{We}$ also examined the expression profile of microRNAs (miRs), which is recently reported to be involved in the pathogenesis of GI disorders including cancers. ${ }^{32,33}$ In $H$. pylori-infected mice, the muscle-specific miRs such as miR-1, miR-133a and miR-133b were significantly down-regulated in the stomachs with an enhancement of the expression of histone deacetylase 4 and serum response factor, which are reported target genes of $m i R-1$ and $m i R-133$ and to enhance the muscular hyperproliferation. Accelerated gastric emptying may be possibly through the disturbed gastric accommodation due to the alterations in muscular layer modification, providing a novel insight into the molecular pathogenesis of dyspepsia associated with $H$. pylori infection. ${ }^{31}$

There might be a reason to consider $H$. pylori-associated dyspepsia as an organic disease and to deal with it as a different disease entity from FD. ${ }^{34,35}$ A new classification based on the pathophysiology and specific symptoms needs to be considered to further the diagnostic and therapeutic advances in this field.

\section{Racial Differences in the Epidemiology of Irritable Bowel Syndrome and Dyspepsia}

There is a paucity of data on racial differences in the epidemiology of IBS alone and UID alone as compared to that of the “overlap syndrome." According to a random survey of 990 subjects who completed a questionnaire which included the Rome II criteria for IBS and FD, ${ }^{35}$ among African Americans, the prevalences of IBS alone, UID alone and their overlap were $0.6 \%$, $17 \%$ and $7.3 \%$, respectively, while among Caucasian Americans, they were $0 \%, 13 \%$ and $13 \%$, respectively. All but 4 patients with IBS had UID. Among patients with UID, overlap syndrome was seen in $30 \%$ of African Americans, as compared to $50 \%$ of Caucasian Americans. African Americans below the poverty line were more likely to have UID than overlap (22\% vs $10 \%$ ). As compared to African Americans, Caucasians with overlap syndrome were likely to be married and to be living in an urban area. There was a higher prevalence of overlap syndrome (UID-IBS) among Caucasians with lower levels of education. UID-IBS overlap was 2.5 times more likely to occur among Caucasians than among African Americans. ${ }^{36}$ However, among UID patients, there might be considerable numbers of patients with peptic ulcer disease or erosive esophagitis.

\section{Conclusion}

The concept of FD-IBS overlap syndrome is important for determining the independent therapeutic approach for such disease conditions of FD alone or of IBS alone, by treating them as a disease of the entire gut as a single organ. Under such concept of disease overlap, FD and IBS can be recognized as being frequently coexisting and not mutually exclusive syndromes.

\section{References}

1. Ford AC, Marwaha A, Lim A, Moayyedi P. Systematic review and meta-analysis of the prevalence of irritable bowel syndrome in individuals with dyspepsia. Clin Gastroenterol Hepatol 2010;8:401409.

2. Kaji M, Fujiwara Y, Shiba M, et al. Prevalence of overlaps between GERD, FD and IBS and impact on health-related quality of life. J Gastroenterol Hepatol 2010;25:1151-1156.

3. Nakajima S, Takahashi K, Sato J, et al. Spectra of functional gastrointestinal disorders diagnosed by Rome III integrative questionnaire in a Japanese outpatient office and the impact of overlapping. J 
Gastroenterol Hepatol 2010;25(suppl 1):S138-S143.

4. Wang A, Liao X, Xiong L, et al. The clinical overlap between functional dyspepsia and irritable bowel syndrome based on Rome III criteria. BMC Gastroenterol 2008;8:43.

5. Lee HJ, Lee SY, Kim JH, et al. Depressive mood and quality of life in functional gastrointestinal disorders: differences between functional dyspepsia, irritable bowel syndrome and overlap syndrome. Gen Hosp Psychiatry 2010;32:499-502.

6. Park H. Functional gastrointestinal disorders and overlap syndrome in Korea. J Gastroenterol Hepatol 2011;26(suppl 3):12-14.

7. Aro P, Talley NJ, Agréus L, et al. Functional dyspepsia impairs quality of life in the adult population. Aliment Pharmacol Ther 2011; 33:1215-1224.

8. Hori K, Matsumoto T, Miwa H. Analysis of the gastrointestinal symptoms of uninvestigated dyspepsia and irritable bowel syndrome. Gut Liver 2009;3:192-196.

9. Corsetti M, Caenepeel P, Fischler B, Janssens J, Tack J. Impact of coexisting irritable bowel syndrome on symptoms and pathophysiological mechanisms in functional dyspepsia. Am J Gastroenterol 2004;99:1152-1159.

10. Suzuki H, Matsuzaki J, Asakura K, et al. High prevalence of lower gastrointestinal (GI) symptoms in functional dyspepsia: a factor and cluster analysis in the general population [abstract]. Gastroenterology 2010;138:S-385.

11. Hongo M. Epidemiology of FGID symptoms in Japanese general population with reference to life style. J Gastroenterol Hepatol 2011;26(suppl 3):19-22.

12. Tack J, Talley NJ, Camilleri M, et al. Functional gastroduodenal disorders. Gastroenterology 2006;130:1466-1479.

13. Noh YW, Jung HK, Kim SE, Jung SA. Overlap of erosive and non-erosive reflux diseases with functional gastrointestinal disorders according to Rome III criteria. J Neurogastroenterol Motil 2010; 16:148-156.

14. Savas LS, White DL, Wieman M, et al. Irritable bowel syndrome and dyspepsia among women veterans: prevalence and association with psychological distress. Aliment Pharmacol Ther 2009;29:115125.

15. Van Oudenhove L, Vandenberghe J, Geeraerts B, et al. Determinants of symptoms in functional dyspepsia: gastric sensorimotor function, psychosocial factors or somatisation? Gut 2008;57:1666-1673.

16. Kindt S, Van Oudenhove L, Mispelon L, Caenepeel P, Arts J, Tack $\mathrm{J}$. Longitudinal and cross-sectional factors associated with long-term clinical course in functional dyspepsia: a 5-year follow-up study. Am J Gastroenterol 2011;106:340-348.

17. Cremon C, Gargano L, Morselli-Labate AM, et al. Mucosal immune activation in irritable bowel syndrome: gender-dependence and association with digestive symptoms. Am J Gastroenterol 2009;104: 392-400.

18. Walker MM, Talley NJ, Prabhakar M, et al. Duodenal mastocytosis, eosinophilia and intraepithelial lymphocytosis as possible disease markers in the irritable bowel syndrome and functional dyspepsia. Aliment Pharmacol Ther 2009;29:765-773.

19. Tack J, Demedts I, Dehondt G, et al. Clinical and pathophysiological characteristics of acute-onset functional dyspepsia. Gastroenterology
2002;122:1738-1747.

20. Mearin F, Pérez-Oliveras M, Perelló A, et al. Dyspepsia and irritable bowel syndrome after a Salmonella gastroenteritis outbreak: one-year follow-up cohort study. Gastroenterology 2005;129:98-104.

21. Porter CK, Gormley R, Tribble DR, Cash BD, Riddle MS. The incidence and gastrointestinal infectious risk of functional gastrointestinal disorders in a healthy US adult population. Am J Gastroenterol 2011;106:130-138.

22. Kindt S, Tertychnyy A, de Hertogh G, Geboes K, Tack J. Intestinal immune activation in presumed post-infectious functional dyspepsia. Neurogastroenterol Motil 2009;21:832-e56.

23. Suzuki H. Post-infectious functional dyspepsia - a novel disease entity among functional gastrointestinal disorders - relation to Helicobacter pylori infection? (Neurogastroenterol Motil 2009;21:832- e56). J Neurogastroenterol Motil 2010;16:97-98.

24. Warren JR, Marshall BJ. Unidentified curved bacilli on gastric epithelium in active chronic gastritis. Lancet 1983;1:1273-1275.

25. Suzuki H, Hibi T, Marshall BJ. Helicobacter pylori: present status and future prospects in Japan. J Gastroenterol 2007;42:1-15.

26. Suzuki H, Iwasaki E, Hibi T. Helicobacter pylori and gastric cancer. Gastric Cancer 2009;12:79-87.

27. Suzuki H, Nishizawa T, Hibi T. Helicobacter pylori eradication therapy. Future Microbiol 2010;5:639-648.

28. Suzuki H, Nishizawa T, Hibi T. Therapeutic strategies for functional dyspepsia and the introduction of the Rome III classification. J Gastroenterol 2006;41:513-523.

29. Moayyedi P, Deeks J, Talley NJ, Delaney B, Forman D. An update of the Cochrane systematic review of Helicobacter pylori eradication therapy in nonulcer dyspepsia: resolving the discrepancy between systematic reviews. Am J Gastroenterol 2003;98:2621-2626.

30. Gwee KA, Teng L, Wong RK, Ho KY, Sutedja DS, Yeoh KG. The response of Asian patients with functional dyspepsia to eradication of Helicobacter pylori infection. Eur J Gastroenterol Hepatol 2009;21: 417-424.

31. Saito Y, Suzuki H, Tsugawa H, et al. Dysfunctional gastric emptying with down-regulation of muscle-specific microRNAs in Helicobacter pylori-infected mice. Gastroenterology 2011;140:189-198.

32. Saito Y, Suzuki H, Hibi T. The role of microRNAs in gastrointestinal cancers. J Gastroenterol 2009;44(suppl 19):18-22.

33. Suzuki H, Saito Y, Hibi T. Chapter 5: MicroRNAs in gastric cancer. In: Cho WCS, ed. MicroRNAs in Cancer Translational Research. Springer 2011:135-143.

34. Suzuki H, Nishizawa T, Hibi T. Can Helicobacter pylori-associated dyspepsia be categorized as functional dyspepsia? J Gastroenterol Hepatol 2011;26(suppl 3):42-45.

35. Suzuki H, Matsuzaki J, Hibi T. What is the difference between Helicobacter pylori-associated dyspepsia and functional dyspepsia? J Neurogastroenterol Motil 2011;17:124-130.

36. Minocha A, Chad W, Do W, Johnson WD. Racial differences in epidemiology of irritable bowel syndrome alone, un-investigated dyspepsia alone, and "overlap syndrome" among African Americans compared to Caucasians: a population-based study. Dig Dis Sci 2006;51:218-226. 